\title{
Molecular dynamics of the cryo-EM CFTR structure
}

Hedvig Tordai ${ }^{\mathrm{a}}$, Ibolya Leveles ${ }^{\mathrm{b}}$, Tamás Hegedüs ${ }^{\mathrm{a}, \mathrm{c}^{*}}$

${ }^{\mathrm{a} D e p a r t m e n t ~ o f ~ B i o p h y s i c s ~ a n d ~ R a d i a t i o n ~ B i o l o g y, ~ S e m m e l w e i s ~ U n i v e r s i t y, ~ B u d a p e s t, ~ H u n g a r y ~}$

${ }^{b}$ Department of Applied Biotechnology and Food Science, Budapest University of Technology and Economics, Budapest, Hungary

${ }^{c}$ Membrane Research Group, Hungarian Academy of Sciences, Budapest, Hungary

*Corresponding author: hegedus.tamas@hegelab.org 


\begin{abstract}
Cystic fibrosis (CF), a lethal monogenic disease, is caused by mutant variants of the $\mathrm{CF}$ transmembrane conductance regulator (CFTR). Recent advances in single molecule cryo-EM methods enabled structural determination of full-length human and zebrafish CFTR, achieving an important milestone for CF drug development. To relate these structures to the gating cycle, we examined its dynamic features using molecular dynamics simulations. Our results show that the nucleotide binding domains (NBDs) in this bottom-open apo conformation exhibit motions related to dimerization and the bottom-closed apo CFTR model indicates opening of NBDs in contrast to transporters. These observations help in understanding the properties of CFTR chloride channel distinct from transporters and in proper interpretation of available structural information on this $\mathrm{ABC}$ protein.
\end{abstract}

Keywords: cystic fibrosis, CFTR, transmembrane protein structure, computational biology, molecular dynamics

\begin{abstract}
Abbreviations: CFTR, cystic fibrosis transmembrane conductance regulator; NBD, nucleotide binding domain; MD, molecular dynamics; TH, transmembrane helix; TMD, transmembrane domain; zfCFTR, zebra fish CFTR
\end{abstract}




\section{Introduction}

Cystic fibrosis (CF) is caused by mutations in the cystic fibrosis transmembrane conductance regulator (CFTR) protein resulting in the lack of functional expression of this chloride channel in the apical membrane of epithelial cells [1]. Intense efforts have been made to correct the basic defect and restore the functional expression of the mutant by small molecules [2,3,4]. However, drug development is still hampered by lack of high resolution CFTR structures.

CFTR is a member of the ABC protein superfamily providing an ion conductance pathway through the cell membrane by two TMD consisting of 6 transmembrane (TM) helices each $[5,6]$. It possesses two nucleotide binding domains (NBDs), which bind ATP, form a transient interaction, and contribute strictly to regulate the channel gating and chloride ions flow along the electrochemical gradient $[7,8]$. The two composite ATP-binding sites are formed by complementary parts of the two NBDs at their domain-domain interface including the Walker $\mathrm{A}$, Walker $\mathrm{B}$ and $\mathrm{ABC}$ signature sequences. [9]. ATP binding alone is not sufficient for channel opening, but requires the phosphorylation of the $\mathrm{R}$ domain as well [10]. This region is a $\sim 200$ a.a. long disordered segment between NBD1 and TMD2 and includes a large number of PKA and other kinase sites.

The atomic structure of several full length $\mathrm{ABC}$ transporters has been determined. Currently four main conformations of Type I ABC exporter structures can be distinguished: (1) inward-facing, bottom-open (Fig. S1 a,b) and (2) inward-facing, bottom-closed conformations in the absence of ATP (Fig. S1 c); (3) outward-facing, bottom-closed in the presence of ATP (Fig. S1 d) and (4) a conformation closed both at the intracellular and extracellular sides (Fig. S1 e) [6]. Ford et al. have made pioneering work to uncover structural features of CFTR, investigated its domain organization, and structural changes upon phosphorylation and nucleotide binding by electron microscopy $[11,12,13]$. They have observed CFTR to accommodate a bottom-closed conformation in crystals [14]. However, the resolution of these structures is generally low $(8>\AA)$, prohibiting studies for drug development or understand the structural effect of mutations.

The recent CFTR structures determined by single particle electron microscopy exhibit significant improvements in resolution and became the ultimate source of further studies aiming CF therapy. The structures of dephosphorylated zebrafish and human CFTR, which have been determined 
in the absence of ATP $[15,16]$, exhibit a significant distance between the two NBDs and the two halves of the molecule show unexpected asymmetric features. In order to learn the role of these 3D features in CFTR structure and function, we assessed various properties of CFTR models and performed in-depth in silico analysis.

\section{Methods}

Structures. Zebrafish and human CFTR structures derived from EM maps were downloaded from the PDB database: (PDBIDs: 5TSI/5UAR, 5U71/5UAK) [15,16]. We also employed the CFTR homology models generated by Corradi et al. based on the TM287/288, McjD, and Sav1866 templates [17]. We call these models as $\mathrm{CFTR}_{\mathrm{TM} 287 / 288}, \mathrm{CFTR}_{\mathrm{MCJD}}$, and $\mathrm{CFTR}_{\mathrm{SAV} 1866}$, respectively.

Molecular dynamics simulations. The structural models were inserted into lipids using the CHARMM-GUI web interface $[18,19]$. Production runs at $310 \mathrm{~K}$ were performed after energy minimization and equilibration steps using GROMACS with CHARMM36 force field [20,21]. Simulations details can be found in the supplementary material and summarized in Table S1. Analysis was done by GROMACS tools, the MDAnalysis package [22] and in-house Python scripts. We demonstrate conformational changes by the distances between the center of mass of the two NBDs. Since the distance can change many ways and characterizes movements ambiguously, we also calculated the number of contacts formed between amino acids of the NBDs throughout the trajectories.

Electrostatics calculations. The electrostatic property of the protein surface was calculated using the Adaptive Poisson-Boltzmann Solver (APBS) at http://www.poissonboltzmann.org [23,24]. Calculations were done using the default parameters (e.g. PARSE force field, PROPKA pH 7) except that a $0.15 \mathrm{M}$ concentration was set for sodium ( $0.95 \AA$ radius) and chloride (1.81 $\AA$ radius) ions. Visualization was done by the PyMOL APBS plugin (Michael Lerner). Color-coding of the electrostatic surface was set from -5 to $+5 \mathrm{~K}_{\mathrm{b}} \mathrm{T} / \mathrm{e}_{\mathrm{c}}$. This was done for visual comparability with earlier studies [15], and for solvation free energy calculations $\left(\Delta \Delta \mathrm{G}=\Delta \mathrm{G}_{\text {membrane }}-\Delta \mathrm{G}_{\text {water }}\right)$ APBSmem was 
employed with default parameters as above $[25,26]$ and the bilayer location was defined based on OPM.

\section{Results}

The experimental CFTR structure possesses unexpected features. In the last decade the CFTR structure was extensively modeled based on the structure of various $\mathrm{ABC}$ transporters. Although key points of the models, such as the position of the coupling helices, have been experimentally confirmed, there was a doubt, whether the transmembrane helices need to exhibit a significantly different conformation for providing a chloride channel function compared to transport. At the first blush, the zfCFTR and hCFTR structures are different from the currently known conformations, exhibiting a one-sided humped configuration with a main protein axis not parallel with the membrane normal. When these CFTR structures are aligned with an ABC structure exhibiting similarly separated NBDs, such as the structure of mMDR1 (PDBID: 4M1M), the overlap of the secondary structural elements is relatively high (Fig. 1). We found that the humped-looking configuration of zfCFTR and hCFTR is the result of the different orientation of the membrane region compared to other $\mathrm{ABC}$ proteins. When the mMDR1 and CFTR structures are aligned, the planes of the bilayers predicted by the OPM server enclose an angle (Fig.1).

\section{[Fig. 1]}

Although most of the CFTR transmembrane helices overlap with MDR1, some of the differences have been claimed to be important. The TH7 is displaced from its usual position compared to other $\mathrm{ABC}$ structures, facilitated by the unusual break in the TH8 [15] (Fig. 1). Because of this break and the tilting described above, we performed surface electrostatics calculations, which indicate charged areas in the membrane region thus suggest structural perturbations in the transmembrane helices of the dephosphorylated CFTR structures (Fig. 2). We also assessed solvation free energy employing the Adaptive Poisson-Boltzmann Solver for membrane proteins [25]. Per residue and total solvation free energies were calculated for hCFTR, homology models, and the templates of the latters. We compared the solvation of the individual transmembrane helices (Table 1 and Fig. S2). Amino 
acids in transmembrane helices $2,6,8$, and 11 of hCFTR exhibit positive values. The larger values in TH6 in all structures can be contributed to Arg347 and Arg352, which have been shown to participate in the channel path $[27,28]$. The TH8 solvation is negative for all the homology models, while possesses a high positive value for the hCFTR structure. Among the structural models, hCFTR exhibits the highest $(-77 \mathrm{~kJ} / \mathrm{mol}), \mathrm{CFTR}_{\mathrm{TM} 287 / 288}$ possesses the lowest $(-259 \mathrm{~kJ} / \mathrm{mol})$ solvation free energy values, and the $\mathrm{CFTR}_{\mathrm{MCJD}}$ has the most transmembrane helices with negative solvation free energy.

[Fig. 2]

The potential disturbances in the TMDs of the experimental CFTR structure may question the conformation and the interaction of the N-terminal, approx. 80 a.a. long L0/Lasso motive with the TM10-11 helices. Although the Lasso motif is not a linker region in CFTR, because of the very high structural similarity with ABCC1/MRP1 L0 ([29,30] and Supplementary Discussion) we suggest to call this segment in $\mathrm{ABCC}$ proteins as the L0/Lasso motif. This $\mathrm{N}$-terminal region also contains amino acids with high solvation free energy values (Fig. S2). However, electrostatic properties of the transmembrane region indicate a valid arrangement of this L0/Lasso segment in the structure, since it is attached to a hydrophobic spot interrupting the positive ring of the transmembrane domains, located at the depth of the inner bilayer boundary. This ring is formed by positively charged amino acids at the interface region of transmembrane helices according to the positive-inside rule $[31,32]$ and is observable in the CFTR homology models, but not well-defined in the experimental structure (Fig. 2).

The inward-facing CFTR structure tends to close in molecular dynamics simulations. The nonphosphorylated zfCFTR and hCFTR in the absence of ATP exhibit an inward-facing conformation with widely open NBDs. Some level of opening, even larger than that of transporters, is not unexpected, since the unphosphorylated $\mathrm{R}$ domain has been envisioned to exert its inhibitory role by intertwining between the NBDs [33]. However, this intertwining could also be realized in the case of the bottom-closed TM287/288-like conformation with partially opened domains (Supplementary Discussion and Figures S3 and S4). It has been claimed for supporting the large NBD separation in hCFTR, that reaction of MTS reagents with cysteine residues engineered into the $\mathrm{ABC}$ signature 
sequence requires a large separation $[15,34]$. While these residues are buried in the ATP-bound NBD1-2 dimer, they are solvent exposed in the bottom-closed apo structure. We demonstrate their accessibility by MTS reagents using in silico docking simulations (Fig. S5).

We assessed the conformation and dynamics of the NBDs in different CFTR structural models using molecular dynamics simulations, which have been implicated as useful tools for checking anomalies in membrane protein structures [35]. Serious changes in the structure, including large rigid body motions and loss of secondary structure has been reported to be observed even in short ( $20 \mathrm{~ns}$ long) simulations in the case of non-native contacts and elements in experimentally determined structures $[35,36]$. In spite of the expectations, based on our earlier simulations with inward-facing structures ([36] and unpublished), not all the simulations with the bottom-open zfCFTR exhibited closing of the NBDs (Fig. 3). One of them remained opened and no interaction is formed between the two NBDs.

\section{[Fig. 3]}

The cryo-EM 3D single particle analysis could not resolve structural heterogeneity and all the particles were included for final reconstruction $[15,16]$. This homogeneity suggests that the hCFTR structure is conformationally biased, especially when the highly dynamic nature of ABC proteins is taken into account [37]. One possibility is that embedding the CFTR in detergent micelle during purification promotes the open conformation of the channel. In order to test the potential effect of a micelle system on the distance between the two NBDs, we created a micelle around zfCFTR and performed MD simulations (Fig. S6). In this system we could observe a delay in closing motions when compared to a lipid bilayer system. However, even simulations with non-contacting NBDs exhibit motions for closing revealed by essential dynamics analysis (Fig. S7).

In order to further test the probability of the bottom-open CFTR conformation, we performed similar simulations with the inward-facing, bottom-closed apo $\mathrm{CFTR}_{\mathrm{TM} 287 / 288}$ model [17]. In two out of six simulations the distance between the NBDs increased and approximated the value observed in the zfCFTR structure, with no contacts between the two domains (Fig. 3). This was unexpected, as we have observed only closing NBD motions in the MDR1 $1_{\mathrm{TM} 287 / 288}$ model (Fig. 3). To assess whether the increased dynamics of the intracellular domains is characteristic for CFTR, we performed simulations 
with the TM287/288 structure embedded in a bilayer. None of the simulations exhibited full opening of the NBDs (Fig. S8).

These results suggest that in addition to the potential promotion of the inward-facing conformation in the presence of the $\mathrm{R}$ domain and the micelle environment, repulsion and opening up may be intrinsic properties of CFTR NDBs that contributes to channel deactivation by exposing a volume for the intrusion of the dephosphorylated R domain. However, the structure widely open at the bottom is most likely not highly populated under physiological conditions as indicated by its exerted tendency to close.

\section{Discussion}

The determination of the CFTR protein structure is an important milestone in cystic fibrosis research. However, the features of the current experimentally determined structures limit the understanding of the gating mechanism and the effect of mutations (Supplementary Discussion). The limitations of single molecule structure determination methods should be taken into account in the case of any application of the accompanying all atom models. E.g. the relative low resolution of certain critical CFTR regions does not allow an accurate determination of amino acid side chain orientation sufficient for drug development. The large distance between the NBDs of the novel CFTR structures draw our attention to the transmembrane domain conformation, which turned out to exhibit largely different angle enclosed with the membrane normal when compared to other $\mathrm{ABC}$ structures. The calculated solvation free energy also revealed larger values than those of CFTR homology models and other structures.

Beside the humped conformation and a potential distortion in the TM regions, the structural homogeneity of CFTR single particles implies that this conformation is highly stabilized or even forced by some factors under experimental conditions (e.g. the micelle, the support grid [38]). We demonstrate that the inward-facing, bottom-open CFTR structure exhibits some level of stability in molecular dynamics simulations and NBDs of the inward-facing, bottom-closed CFTR $\mathrm{TM} 287 / 288_{1}$ can 
open up. These observations suggest that the bottom-open state is populated at a low probability under physiological conditions and may not be required for the regulation and function of CFTR. We show that a smaller space between the NBDs in the $\mathrm{CFTR}_{\mathrm{TM} 287 / 288}$ conformation is sufficient for events (e.g. accessibility of MTS reagents, intertwining of R domain) which have been suggested to need a widely open conformation.

It is important to emphasize that the semi-high resolution structure of the non-phosphorylated zfCFTR and hCFTR together with the low resolution structure of the phosphorylated and bottom closed CFTR do not reconcile a chloride channel pathway for the active state $[14,15,16]$ (Fig. 4 and Supplementary Discussion). Several pore-forming residues (Fig. 4, colored by red and blue) and salt bridges stabilizing the conducting state are close to each other in the dephosphorylated structure but largely separated in the bottom-closed model. Green residues have been discussed as amino acids participating in recruitment of negative chloride ions at the extracellular sides. This conclusion may not be strong, since some of the yellow residues, which do not influence the properties of the chloride channel, have similar spatial localization and could also recruit negative ions. Since both conformations are compatible with some functional data, we presume that both types of conformations possess physiological features, but represent short-living conformations under physiological conditions. Similar to Corradi et al. [17] we suggest that the conducting conformation is similar to that of McjD with TM helices close to each other both at the bottom and the top (Fig. 4 c). Importantly, CFTR $_{\text {MCID }}$ exhibits pathways with a lateral opening at the cytoplasmic loops and openings between the extracellular loops. The lateral opening of the presented pathway located between TM4 and TM6 was also detected in MD simulations by Mornon et al. [39,40] and incorporates K370 affecting channel properties. While the published experimental structures are undoubtedly important in the heroic work of deciphering structure/function relationships of CFTR, there is a high demand to gather further data on various conformers at a higher resolution and possibly under more physiological conditions (e.g. in nanodisc). Till then the field is highly dependent on various models built based either on the published electron densities or on homology.

In the last decade CFTR homology models were used extensively to guide structure-function studies as discussed in a recent review by Callebaut et al. [41]. Since the homology models rely on 
multiple sequence alignments and using numerous experimental constraints, in principle, they are expected to be similar to experimental structures in many specific features. Since several properties of the inward-facing structure are unusual and may present a sparsely populated conformation under physiological conditions, the CFTR homology models of various conformations and their proper utilization in CFTR research are still crucial.

Our work also highlights the importance of complex in silico analysis of structural data on transmembrane proteins. Measures used for validate the quality of experimental structures of soluble proteins (e.g. ratio of disallowed dihedral angles) should be supplemented with additional methods assessing various properties of transmembrane regions. Taking additional measures into account for structure validation are likely important not only for CFTR, but for every membrane proteins.

\section{Acknowledgments}

Thanks for helpful discussions to Gergely Lukacs, McGill University, Montreal. This work was supported by the Bolyai Fellowship of the Hungarian Academy of Sciences (TH), NKFIH K 111678 (TH). We acknowledge NIIF and Wigner GPU Laboratory for awarding us access to resources based in Hungary, and the support of their stuff is gratefully acknowledged.

\section{References}

[1] J.R. Riordan, J.M. Rommens, B. Kerem, N. Alon, R. Rozmahel, Z. Grzelczak, J. Zielenski, S. Lok, N. Plavsic, J.L. Chou, et al., Identification of the cystic fibrosis gene: cloning and characterization of complementary DNA, Science 245 (1989) 1066-1073.

[2] G. Veit, R.G. Avramescu, D. Perdomo, P.W. Phuan, M. Bagdany, P.M. Apaja, F. Borot, D. Szollosi, Y.S. Wu, W.E. Finkbeiner, T. Hegedus, A.S. Verkman, G.L. Lukacs, Some gating potentiators, including VX-770, diminish DeltaF508-CFTR functional expression, Sci Transl Med 6 (2014) 246 ra297.

[3] D.M. Cholon, N.L. Quinney, M.L. Fulcher, C.R. Esther, Jr., J. Das, N.V. Dokholyan, S.H. Randell, R.C. Boucher, M. Gentzsch, Potentiator ivacaftor abrogates pharmacological correction of DeltaF508 CFTR in cystic fibrosis, Sci Transl Med 6 (2014) 246 ra296.

[4] M.P. Boyle, S.C. Bell, M.W. Konstan, S.A. McColley, S.M. Rowe, E. Rietschel, X. Huang, D. Waltz, N.R. Patel, D. Rodman, A CFTR corrector (lumacaftor) and a CFTR potentiator (ivacaftor) for treatment of patients with cystic fibrosis who have a phe508del CFTR mutation: a phase 2 randomised controlled trial, Lancet Respir Med 2 (2014) 527-538.

[5] J. ter Beek, A. Guskov, D.J. Slotboom, Structural diversity of ABC transporters, J Gen Physiol 143 (2014) 419-435.

[6] K.P. Locher, Mechanistic diversity in ATP-binding cassette (ABC) transporters, Nat Struct Mol Biol 23 (2016) 487-493. 
[7] N.A. McCarty, Permeation through the CFTR chloride channel, J Exp Biol 203 (2000) 1947-1962.

[8] D. Muallem, P. Vergani, Review. ATP hydrolysis-driven gating in cystic fibrosis transmembrane conductance regulator, Philos Trans R Soc Lond B Biol Sci 364 (2009) 247-255.

[9] R.J. Dawson, K.P. Locher, Structure of a bacterial multidrug ABC transporter, Nature 443 (2006) 180-185.

[10] S.H. Cheng, D.P. Rich, J. Marshall, R.J. Gregory, M.J. Welsh, A.E. Smith, Phosphorylation of the R domain by cAMP-dependent protein kinase regulates the CFTR chloride channel, Cell 66 (1991) 1027-1036.

[11] L. Zhang, L.A. Aleksandrov, J.R. Riordan, R.C. Ford, Domain location within the cystic fibrosis transmembrane conductance regulator protein investigated by electron microscopy and gold labelling, Biochim Biophys Acta 1808 (2011) 399-404.

[12] L. Zhang, L.A. Aleksandrov, Z. Zhao, J.R. Birtley, J.R. Riordan, R.C. Ford, Architecture of the cystic fibrosis transmembrane conductance regulator protein and structural changes associated with phosphorylation and nucleotide binding, J Struct Biol 167 (2009) 242-251.

[13] N. Cant, N. Pollock, R.C. Ford, CFTR structure and cystic fibrosis, Int J Biochem Cell Biol 52 (2014) 15-25.

[14] M.F. Rosenberg, L.P. O'Ryan, G. Hughes, Z. Zhao, L.A. Aleksandrov, J.R. Riordan, R.C. Ford, The cystic fibrosis transmembrane conductance regulator (CFTR): three-dimensional structure and localization of a channel gate, J Biol Chem 286 (2011) 42647-42654.

[15] Z. Zhang, J. Chen, Atomic Structure of the Cystic Fibrosis Transmembrane Conductance Regulator, Cell 167 (2016) 1586-1597 e1589.

[16] F. Liu, Z. Zhang, L. Csanady, D.C. Gadsby, J. Chen, Molecular Structure of the Human CFTR Ion Channel, Cell 169 (2017) 85-95 e88.

[17] V. Corradi, P. Vergani, D.P. Tieleman, Cystic Fibrosis Transmembrane Conductance Regulator (CFTR): CLOSED AND OPEN STATE CHANNEL MODELS, J Biol Chem 290 (2015) 22891-22906.

[18] E.L. Wu, X. Cheng, S. Jo, H. Rui, K.C. Song, E.M. Davila-Contreras, Y. Qi, J. Lee, V. Monje-Galvan, R.M. Venable, J.B. Klauda, W. Im, CHARMM-GUI Membrane Builder toward realistic biological membrane simulations, J Comput Chem 35 (2014) 1997-2004.

[19] J. Lee, X. Cheng, J.M. Swails, M.S. Yeom, P.K. Eastman, J.A. Lemkul, S. Wei, J. Buckner, J.C. Jeong, Y. Qi, S. Jo, V.S. Pande, D.A. Case, C.L. Brooks, 3rd, A.D. MacKerell, Jr., J.B. Klauda, W. Im, CHARMM-GUI Input Generator for NAMD, GROMACS, AMBER, OpenMM, and CHARMM/OpenMM Simulations Using the CHARMM36 Additive Force Field, J Chem Theory Comput 12 (2016) 405-413.

[20] S. Pronk, S. Pall, R. Schulz, P. Larsson, P. Bjelkmar, R. Apostolov, M.R. Shirts, J.C. Smith, P.M. Kasson, D. van der Spoel, B. Hess, E. Lindahl, GROMACS 4.5: a high-throughput and highly parallel open source molecular simulation toolkit, Bioinformatics 29 (2013) 845-854.

[21] R.B. Best, X. Zhu, J. Shim, P.E.M. Lopes, J. Mittal, M. Feig, A.D. MacKerell, Optimization of the Additive CHARMM All-Atom Protein Force Field Targeting Improved Sampling of the Backbone phi, psi and Side-Chain chi(1) and chi(2) Dihedral Angles, Journal of Chemical Theory and Computation 8 (2012) 3257-3273.

[22] N. Michaud-Agrawal, E.J. Denning, T.B. Woolf, O. Beckstein, MDAnalysis: a toolkit for the analysis of molecular dynamics simulations, J Comput Chem 32 (2011) 2319-2327.

[23] N.A. Baker, D. Sept, S. Joseph, M.J. Holst, J.A. McCammon, Electrostatics of nanosystems: application to microtubules and the ribosome, Proc Natl Acad Sci U S A 98 (2001) 1003710041.

[24] T.J. Dolinsky, P. Czodrowski, H. Li, J.E. Nielsen, J.H. Jensen, G. Klebe, N.A. Baker, PDB2PQR: expanding and upgrading automated preparation of biomolecular structures for molecular simulations, Nucleic Acids Res 35 (2007) W522-525.

[25] K.M. Callenberg, O.P. Choudhary, G.L. de Forest, D.W. Gohara, N.A. Baker, M. Grabe, APBSmem: a graphical interface for electrostatic calculations at the membrane, PLoS One 5 (2010).

[26] D. Sitkoff, K.A. Sharp, B. Honig, Accurate calculation of hydration free energies using macroscopic solvent models, Journal of Physical Chemistry 98 (1994) 1978-1988. 
[27] J.F. Cotten, M.J. Welsh, Cystic fibrosis-associated mutations at arginine 347 alter the pore architecture of CFTR. Evidence for disruption of a salt bridge, J Biol Chem 274 (1999) 54295435.

[28] P. Linsdell, Architecture and functional properties of the CFTR channel pore, Cell Mol Life Sci 74 (2017) 67-83.

[29] Z.L. Johnson, J. Chen, Structural Basis of Substrate Recognition by the Multidrug Resistance Protein MRP1, Cell 168 (2017) 1075-1085 e1079.

[30] E. Bakos, R. Evers, G. Szakacs, G.E. Tusnady, E. Welker, K. Szabo, M. de Haas, L. van Deemter, P. Borst, A. Varadi, B. Sarkadi, Functional multidrug resistance protein (MRP1) lacking the Nterminal transmembrane domain, J Biol Chem 273 (1998) 32167-32175.

[31] A. Elazar, J.J. Weinstein, J. Prilusky, S.J. Fleishman, Interplay between hydrophobicity and the positive-inside rule in determining membrane-protein topology, Proc Natl Acad Sci U S A 113 (2016) 10340-10345.

[32] G. von Heijne, Control of topology and mode of assembly of a polytopic membrane protein by positively charged residues, Nature 341 (1989) 456-458.

[33] Z. Bozoky, M. Krzeminski, R. Muhandiram, J.R. Birtley, A. Al-Zahrani, P.J. Thomas, R.A. Frizzell, R.C. Ford, J.D. Forman-Kay, Regulatory R region of the CFTR chloride channel is a dynamic integrator of phospho-dependent intra- and intermolecular interactions, Proc Natl Acad Sci U S A 110 (2013) E4427-4436.

[34] L.A. Chaves, D.C. Gadsby, Cysteine accessibility probes timing and extent of NBD separation along the dimer interface in gating CFTR channels, J Gen Physiol 145 (2015) 261-283.

[35] A. Ivetac, M.S. Sansom, Molecular dynamics simulations and membrane protein structure quality, Eur Biophys J 37 (2008) 403-409.

[36] G. Gyimesi, S. Ramachandran, P. Kota, N.V. Dokholyan, B. Sarkadi, T. Hegedus, ATP hydrolysis at one of the two sites in $A B C$ transporters initiates transport related conformational transitions, Biochim Biophys Acta 1808 (2011) 2954-2964.

[37] A. Moeller, S.C. Lee, H. Tao, J.A. Speir, G. Chang, I.L. Urbatsch, C.S. Potter, B. Carragher, Q. Zhang, Distinct conformational spectrum of homologous multidrug $A B C$ transporters, Structure 23 (2015) 450-460.

[38] R.F. Thompson, M. Walker, C.A. Siebert, S.P. Muench, N.A. Ranson, An introduction to sample preparation and imaging by cryo-electron microscopy for structural biology, Methods 100 (2016) 3-15.

[39] J.P. Mornon, B. Hoffmann, S. Jonic, P. Lehn, I. Callebaut, Full-open and closed CFTR channels, with lateral tunnels from the cytoplasm and an alternative position of the F508 region, as revealed by molecular dynamics, Cell Mol Life Sci 72 (2015) 1377-1403.

[40] J.P. Mornon, P. Lehn, I. Callebaut, Molecular models of the open and closed states of the whole human CFTR protein, Cell Mol Life Sci 66 (2009) 3469-3486.

[41] I. Callebaut, B. Hoffmann, P. Lehn, J.P. Mornon, Molecular modelling and molecular dynamics of CFTR, Cell Mol Life Sci 74 (2017) 3-22.

\section{Figure legends}

Fig. 1 Comparison of the membrane orientation of zfCFTR and mMDR1. (a) hCFTR (PDBID: 5UAK) and mMDR1 (PDBID: 4M1M; gray) were structurally aligned and their transmembrane region are labeled with two layers of dots (blue and black, respectively), determined using the OPM server. (b) There is an unexpected break in TM8 (blue) of zfCFTR (shown here) and hCFTR. TMDs: green, cyan, NBDs: orange, yellow, TH8 segment: blue. 
Fig. 2 Electrostatics calculations suggest disturbance in the TM helices, while a proper binding location of the L0/Lasso region. (a) Calculations performed by the APBS server (Adaptive PoissonBolzman Solver and displayed in PyMOL) indicate charged residues of zfCFTR exposed to the hydrophobic part of the bilayer (arrows). (b) The same calculation is shown also for mMDR1. (c) Electrostatics at the protein surface underlines the location of the intramolecular interaction of the

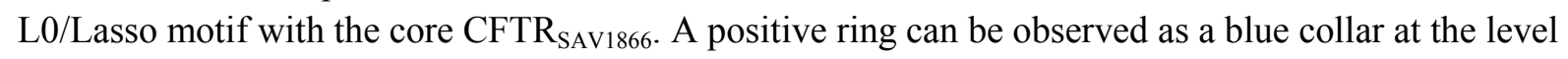
of the membrane inner interface disrupted by a hydrophobic patch (white) at the location of L0/Lasso region binding (TH10-11; arrow). Approximate bilayer boundaries are indicated by dashed lines.

Fig. 3 zfCFTR in a bilayer is biased towards NBD closure, while CFTR $_{\text {TM287/288 }}$ opens up in MD simulations. Six simulations were performed with zfCFTR (PDBID: 5UAR) embedded in a POPC bilayer. (a) The distance of the center of mass of NBDs is shown in the top panel. The bottom panel shows the number of amino acid contacts $(\mathrm{C} \alpha-\mathrm{C} \alpha<=8 \AA$ ) between the NBDs throughout the $50 \mathrm{~ns}$ long simulations. Six simulations were performed with both $\mathrm{CFTR}_{\mathrm{TM} 287 / 288}(\mathbf{b})$ and $\mathrm{MDR}_{\mathrm{TM} 287 / 288}(\mathbf{c})$ in a POPC bilayer. In two cases (black, red) the NBDs in $\mathrm{CFTR}_{\mathrm{TM} 287 / 288}$ got farther from each other and reached a distance as in zfCFTR (solid horizontal line), losing all the contacts between them. In the case of the MDR1 model the NBDs tended to form more contacts. The horizontal lines show this value for the $\mathrm{CFTR}_{\mathrm{TM} 287 / 288}$ (solid) and the CFTR $\mathrm{SAV} 1866_{\text {(dotted) homology models and the initial }}$ conformation of zfCFTR (dashed) as references.

Fig. 4 It is challenging to integrate the atomic level data into the gating cycle. Inward-facing hCFTR (a; PDBID: 5UAK), outward-facing CFTR $_{\mathrm{SAV}_{1866}}(\mathbf{b})$, and $\mathrm{CFTR}_{\mathrm{MCJD}}$ model (c) are shown in gray. Specific positively charged residues are shown in stick representation and colored as follows. Pore constriction residues (colored by red) are close to each other in the non-phosphorylated (a) and McjD-based (c) structures. Blue residues surrounding the large vestibule participate in constructing chloride pathway. Green residues have been indicated as amino acids participating in recruitment of negative chloride ions at the extracellular side. Cyan residues show the lateral openings suggested by MD simulations. Purple is one of the detected pathways. 
Table 1 Solvation free energy of CFTR structural models calculated by APBSmem

\begin{tabular}{|c|c|c|c|c|}
\hline & \multicolumn{4}{|c|}{ Solvation free energy $(\mathrm{kJ} / \mathrm{mol})$} \\
\hline & hCFTR & $\mathrm{CFTR}_{\text {TM287/288 }}$ & CFTR $_{\text {MCJD }}$ & CFTR $_{\text {SAV1866 }}$ \\
\hline templates* & & -226 & -488 & -267 \\
\hline full length ${ }^{* *}$ & -77 & -259 & -226 & -224 \\
\hline TH1 & -14 & 28 & -18 & 3 \\
\hline TH2 & 2 & -29 & -27 & -81 \\
\hline TH3 & -22 & -40 & -32 & -63 \\
\hline TH4 & -90 & -107 & -87 & -108 \\
\hline TH5 & -1 & -50 & -30 & 35 \\
\hline TH6 & 9 & 73 & 26 & 13 \\
\hline TH7 & -61 & -84 & -33 & -73 \\
\hline TH8 & 61 & -40 & -34 & -27 \\
\hline TH9 & -18 & -51 & -41 & -4 \\
\hline TH10 & -102 & -84 & -77 & -84 \\
\hline TH11 & 51 & 50 & -5 & 34 \\
\hline TH12 & -9 & -18 & -19 & -24 \\
\hline
\end{tabular}

* PDBIDs: 3QF4, 4PLO, and 2HYD

** residues present in all CFTR structures 